\title{
Asymptotic Optimality of Combined Double Sequential Weighted Probability Ratio Test for Three Composite Hypotheses
}

\author{
Lei Wang, Xiaolong Pu, and Yan Li \\ School of Finance and Statistics, East China Normal University, Shanghai 200241, China \\ Correspondence should be addressed to Lei Wang; leiwang.stat@gmail.com
}

Received 24 December 2014; Revised 13 March 2015; Accepted 15 March 2015

Academic Editor: Antonino Laudani

Copyright (C) 2015 Lei Wang et al. This is an open access article distributed under the Creative Commons Attribution License, which permits unrestricted use, distribution, and reproduction in any medium, provided the original work is properly cited.

\begin{abstract}
We propose the weighted expected sample size (WESS) to evaluate the overall performance on the indifference-zones for three composite hypotheses' testing problem. Based on minimizing the WESS to control the expected sample sizes, a new sequential test is developed by utilizing two double sequential weighted probability ratio tests (2-SWPRTs) simultaneously. It is proven that the proposed test has a finite stopping time and is asymptotically optimal in the sense of asymptotically minimizing not only the expected sample size but also any positive moment of the stopping time on the indifference-zones under some mild conditions. Simulation studies illustrate that the proposed test has the smallest WESS and relative mean index (RMI) compared with Sobel-Wald and Whitehead-Brunier tests.
\end{abstract}

\section{Introduction}

Let $X_{1}, X_{2}, \ldots$ be independent and identically distributed (i.i.d.) random variables whose common density function $f(x, \theta)$ (with respect to some nondegenerate measure $v$ ) belongs to the exponential family

$$
f(x, \theta)=\exp \{x \theta-\psi(\theta)\}, \quad \theta \in \Theta=(\underline{\theta}, \bar{\theta}),
$$

where $\psi(\cdot)$ is a convex function and $\Theta$ is the natural parameter space with $-\infty \leq \underline{\theta}<\bar{\theta} \leq \infty$. The problem of interest is the following three composite hypotheses' testing problem:

$$
\begin{aligned}
& H_{1}: \theta \leq \theta_{1} \text { versus } \\
& H_{2}: \theta_{2} \leq \theta \leq \theta_{3} \text { versus } \\
& H_{3}: \theta \geq \theta_{4} \\
& \left(\theta_{1}<\theta_{2}<\theta_{3}<\theta_{4}\right),
\end{aligned}
$$

where $\theta_{1}, \theta_{2}, \theta_{3}, \theta_{4} \in \Theta$. For example, in clinical trial applications, in order to compare the effects of two drugs (Goeman et al. [1]), the equivalence trial $H_{0}:|\Delta|>\Delta_{1}$ versus $H_{1}:|\Delta|<\Delta_{0}\left(0<\Delta_{0}<\Delta_{1}\right)$ would be more realistically stated as $H_{1}: \Delta<-\Delta_{1}$ (inferiority), $H_{2}:-\Delta_{0}<\Delta<\Delta_{0}$ (equivalence), and $H_{3}: \Delta>\Delta_{1}$ (superiority), where $\Delta$ is the difference of effect between two drugs. The sequential testing of three or more hypotheses has been applied to a variety of engineering problems such as pattern recognition (Fu [2]; McMillen and Holmes [3]), multiple-resolution radar detection (Bussgang [4]), products comparisons (Anderson [5]), and others ( $\mathrm{Li}$ et al. [6]). The intervals of $\left[\theta_{1}, \theta_{2}\right]$ and $\left[\theta_{3}, \theta_{4}\right]$ are usually called indifference-zones and denoted by $\bar{\Theta}=\left[\theta_{1}, \theta_{2}\right] \cup\left[\theta_{3}, \theta_{4}\right]$.

Published work on this problem has taken two main approaches. Pavlov [7], Baum and Veeravalli [8], and Dragalin et al. $[9,10]$ studied the class of tests motivated by the Bayesian framework. The second approach has focused on extending the sequential probability ratio test (SPRT) and double sequential probability ratio test (2-SPRT) to incorporate more than two hypotheses, such as Sobel and Wald [11], Armitage [12], Simons [13], Lorden [14], Whitehead and Brunier [15], and Li and Pu [16, 17]. Dragalin and Novikov [18] studied the problem of testing several composite hypotheses with an indifference-zone for an unknown parameter. Lai [19] considered the multihypothesis testing problem where some or all of these hypotheses are composite. 
Among others, the tests proposed by Sobel and Wald [11] and Whitehead and Brunier [15] are usually used in practice for problem (2). Specifically, Sobel and Wald [11] proposed carrying out simultaneous SPRTs of $H_{1}$ versus $\mathrm{H}_{2}$ and $\mathrm{H}_{2}$ versus $\mathrm{H}_{3}$. However, when the true parameter is in the indifference-zones, the expected sample size of the Sobel-Wald test can be considerably larger than that of a fixed-sample-size test plan. Moreover, it is untruncated such that the number of observations required can not be predetermined, an undesirable property in many practical situations such as medical trial. To reduce the maximum expected sample size, Whitehead and Brunier [15] applied two 2-SPRTs instead of two SPRTs for the component tests, at the cost of larger expected sample sizes when the true parameter does not belong to the indifference-zones.

For one-sided composite hypotheses, in order to control the expected sample sizes, Wang et al. [20] proposed the double sequential weighted probability ratio test (2-SWPRT) based on mixture likelihood ratio statistics and showed that the 2-SWPRT is an asymptotically overall optimal test in the sense of asymptotically minimizing the expected sample sizes on the indifference-zone. Motivated by the attractive properties of the 2-SWPRT, we extend the existing work on problem (2) from pointwise optimality to overall performance optimality when there are different concerns of interest on different $\theta$ s. In particular, we propose an optimality criterion to evaluate the overall performance of sequential test plans on the indifference-zones for three composite hypotheses and correspondingly develop a new sequential test for problem (2) by utilizing two 2-SWPRTs as the component tests to reduce the expected sample sizes. We show the proposed test has a finite stopping time and is asymptotically optimal in the sense of asymptotically minimizing not only the expected sample size but also any positive moment of the stopping time on the indifference-zones. Simulation studies show that the proposed test not only has the smallest WESS compared with Sobel-Wald and Whitehead-Brunier tests, but also is superior to the Whitehead-Brunier test and comparable with the Sobel-Wald test when the true parameter does not belong to the indifference-zones. Moreover, the RMI also shows the proposed test is an efficient method to improve the overall performance.

The rest of this paper is organized as follows. In Section 2, we review the Sobel-Wald and Whitehead-Brunier tests. The combined double sequential weighted probability ratio test (denoted by combined 2-SWPRT) is proposed and its properties are given in Section 3. Simulation results are provided in Section 4 and some conclusions are in Section 5. All technical details are given in Appendix.

\section{Methodology Review}

For one-sided composite hypotheses $H_{1}$ versus $H_{2}$, the SPRT is optimal in the sense that it minimizes the expected sample sizes at $\theta_{1}$ and $\theta_{2}$, and the 2-SPRT has (approximately) minimal maximum expected sample size over $\left(\theta_{1}, \theta_{2}\right)$ among all sequential and nonsequential tests with the same error probabilities. Given the well-known optimality properties of the SPRT and 2-SPRT, it is natural to use the SPRTs and
2-SPRTs as the component tests to construct the sequential tests for problem (2), respectively. In this section, we briefly review the Sobel-Wald and Whitehead-Brunier tests.

For testing problem (2), the generalization of errors of types I and II is expressible in terms of a $3 \times 3$ error matrix $E=\left(\alpha_{j k}\right)$, where $\alpha_{j k}=P$ [accepting $H_{j} \mid H_{k}$ is true] for $j, k=1,2,3$. However, under some mild conditions, Sobel and Wald [11, pages 504-505] and Armitage [12, pages 142143] showed that $\alpha_{31}$ and $\alpha_{13}$ are zero, which can be verified by the simulation results in Section 4. It becomes apparent that in the general case we have at most four "degrees of freedom" in choosing an error matrix. Without loss of generality, we consider $\Delta=(\tau, d)$ as a sequential test for problem (2), where $\tau$ is the stopping rule and $d$ is the decision rule $(d=k$ means accepting $\left.H_{k}, k=1,2,3\right)$. Set $\Theta_{1}=\left(\underline{\theta}, \theta_{1}\right], \Theta_{2}=\left[\theta_{2}, \theta_{3}\right]$ and $\Theta_{3}=\left[\theta_{4}, \bar{\theta}\right)$. Given positive vectors $\alpha=\left(\alpha_{1}, \alpha_{2}\right)$ and $\beta=\left(\beta_{1}, \beta_{2}\right)\left(\alpha_{i}+\beta_{i}<1, i=1,2\right)$,

$$
\begin{gathered}
\Upsilon(\alpha, \beta)=\left\{\Delta=(\tau, d): \sup _{\theta \in \Theta_{i}} P(d=i+1) \leq \alpha_{i},\right. \\
\left.\sup _{\theta \in \Theta_{i+1}} P(d=i) \leq \beta_{i}, i=1,2\right\}
\end{gathered}
$$

is the set of all sequential tests with error probabilities controlled by $\alpha$ and $\beta$.

(1) Sobel-Wald Test. Since the hypotheses $H_{1}, H_{2}$, and $H_{3}$ are ordered, the sequential testing of problem (2) can be constructed by combining the following two one-sided composite hypotheses $S_{1}$ and $S_{2}$ :

$$
\begin{aligned}
& S_{1}: \theta \leq \theta_{1} \quad \text { versus } \theta_{2} \leq \theta \leq \theta_{3} \\
& S_{2}: \theta_{2} \leq \theta \leq \theta_{3} \quad \text { versus } \theta \geq \theta_{4} .
\end{aligned}
$$

Sobel and Wald [11] proposed operating $S_{1}$ and $S_{2}$ by the SPRTs simultaneously. For all $\theta, \rho \in \Theta$, define $r_{n}(\theta, \rho)=$ $\prod_{l=1}^{n} f\left(x_{l}, \theta\right) / f\left(x_{l}, \rho\right)$. The stopping and decision rules of $S_{i}(i=1,2)$ determined by the SRPT are

$$
\begin{gathered}
\tau_{i}^{s}=\inf \left\{n \geq 1: r_{n}\left(\theta_{2 i}, \theta_{2 i-1}\right) \notin\left(A_{i}^{s}, B_{i}^{s}\right)\right\}, \quad i=1,2, \\
d_{i}^{s}=I\left(\tau_{i}^{s}<\infty, r_{\tau_{i}^{s}}\left(\theta_{2 i}, \theta_{2 i-1}\right) \geq B_{i}^{s}\right)+i,
\end{gathered}
$$

where $I(\cdot)$ is the indicator function and $A_{i}^{s}$ and $B_{i}^{s}(i=1,2)$ are the boundary parameters $\left(0<A_{i}^{s}<1<B_{i}^{\mathcal{S}}<\infty\right)$, which are usually set as

$$
A_{i}^{s}=\frac{\beta_{i}}{1-\alpha_{i}}, \quad B_{i}^{s}=\frac{1-\beta_{i}}{\alpha_{i}}, \quad i=1,2
$$

to meet requirements on the error probabilities. When $B_{1}^{s} / B_{2}^{s} \leq 1$ and $A_{1}^{s} / A_{2}^{s} \leq 1$, Sobel and Wald [11] showed 
the event $\left\{d_{1}^{s}=1, d_{2}^{s}=3\right\}$ is impossible. The stopping and decision rules of the Sobel-Wald test are defined as

$$
\begin{aligned}
& \tau^{s}=\max \left(\tau_{1}^{s}, \tau_{2}^{s}\right),
\end{aligned}
$$

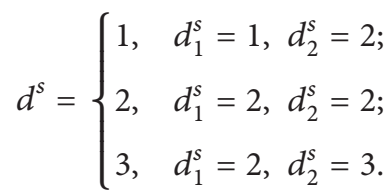

The Sobel-Wald test is optimal in the sense that it minimizes the expected sample sizes at $\theta_{2 i-1}$ and $\theta_{2 i}(i=1,2)$ among all sequential and nonsequential tests whose error probabilities satisfy $\Upsilon(\alpha, \beta)$. However, its expected sample sizes at other parameters over $\bar{\Theta}$ may be unsatisfactory.

(2) Whitehead-Brunier Test. In order to minimize the maximum expected sample size under constraints (3), Whitehead and Brunier [15] applied the 2-SPRT to operate $S_{1}$ and $S_{2}$, instead of the SPRT. As in Lorden [21], let $K(\theta, \rho)=$ $E_{\theta} \log [f(x, \theta) / f(x, \rho)]$ be the Kullback-Leibler (KL) information number. Define $\widehat{\theta}_{i} \in\left(\theta_{2 i-1}, \theta_{2 i}\right)$ and $n_{i}^{*}(i=1,2)$ by

$$
\frac{\left|\log \alpha_{i}\right|}{K\left(\widehat{\theta}_{i}, \theta_{2 i-1}\right)}=\frac{\left|\log \beta_{i}\right|}{K\left(\widehat{\theta}_{i}, \theta_{2 i}\right)}=n_{i}^{*}, \quad i=1,2 .
$$

Set $c_{i}^{*}$ such that $\Phi\left(c_{i}^{*}\right)=-a_{2 i}^{*} /\left(a_{2 i-1}^{*}-a_{2 i}^{*}\right), i=1,2$, where $\Phi(\cdot)$ is the cumulative distribution function of the standard normal distribution, $a_{2 i-1}^{*}=\left(\widehat{\theta}_{i}-\theta_{2 i-1}\right) / K\left(\widehat{\theta}_{i}, \theta_{2 i-1}\right)$, and $a_{2 i}^{*}=$ $\left(\widehat{\theta}_{i}-\theta_{2 i}\right) / K\left(\widehat{\theta}_{i}, \theta_{2 i}\right), i=1,2$. Let

$$
\theta_{i}^{*}=\widehat{\theta}_{i}+c_{i}^{*}\left[n_{i}^{*} \psi^{\prime \prime}\left(\widehat{\theta}_{i}\right)\right]^{-1 / 2}, \quad i=1,2 .
$$

The stopping and decision rules of $S_{i}(i=1,2)$ determined by the 2-SPRT are

$$
\begin{gathered}
\tau_{i}^{w}=\inf \left\{n \geq 1: r_{n}\left(\theta_{i}^{*}, \theta_{2 i-1}\right) \geq A_{i}^{w} \text { or } r_{n}\left(\theta_{i}^{*}, \theta_{2 i}\right) \geq B_{i}^{w}\right\}, \\
i=1,2, \\
d_{i}^{w}=I\left(\tau_{i}^{w}<\infty, r_{\tau_{i}^{w}}\left(\theta_{i}^{*}, \theta_{2 i-1}\right) \geq A_{i}^{w}\right)+i
\end{gathered}
$$

where $A_{i}^{w}$ and $B_{i}^{w}(i=1,2)$ are the boundary parameters $\left(0<A_{i}^{w}, B_{i}^{w}<\infty\right)$. The conservative values of $A_{i}^{w}$ and $B_{i}^{w}$ are $1 / \alpha_{i}$ and $1 / \beta_{i}$, in the sense that the real error probabilities may be much smaller than $\alpha_{i}$ and $\beta_{i}(i=1,2)$, respectively. The stopping and decision rules of the Whitehead-Brunier test are defined as

$$
\begin{gathered}
\tau^{w}=\max \left(\tau_{1}^{w}, \tau_{2}^{w}\right) \\
d^{w}= \begin{cases}1, & d_{1}^{w}=1, d_{2}^{w}=2 \\
2, & d_{1}^{w}=2, \\
3, & d_{2}^{w}=2,\end{cases}
\end{gathered}
$$

\section{Optimality Criterion and Combined 2-SWPRT}

For testing problem (2), if $\theta<\theta_{1}$ we prefer to accept $H_{1}$ and this preference is the stronger the smaller $\theta$. Similarly, if $\theta>\theta_{4}$ we prefer to accept $H_{3}$, and we prefer to accept $H_{2}$ if $\theta_{2}<\theta<\theta_{3}$. However, we have no strong preference between $H_{1}$ and $H_{2}$ if $\theta \in\left[\theta_{1}, \theta_{2}\right]$, and we also have no strong preference between $H_{2}$ and $H_{3}$ if $\theta \in\left[\theta_{3}, \theta_{4}\right]$. In these cases, we need more observations for decision. Thus, when the error probabilities satisfy $\Upsilon(\alpha, \beta)$, we focus on reduction of the expected sample sizes over the indifference-zones $\bar{\Theta}$ in applications. Let $g(\theta)$ be a nonnegative weight function which is sectionally continuous on $\left[\theta_{1}, \theta_{2}\right]$ and $\left[\theta_{3}, \theta_{4}\right]$, respectively, and satisfies $\int_{\bar{\Theta}} g(\theta) d \theta=1$. We define the weighted expected sample size as

$$
\operatorname{WESS}(g)=\int_{\bar{\Theta}} E_{\theta} \tau \cdot g(\theta) d \theta
$$

to evaluate the overall performance of sequential test plans on $\bar{\Theta}$. The choice of $g$ should be chosen according to practical needs (Sobel and Wald [11]). For example, let $g(\theta)$ be uniform weights when there are no differences on $\bar{\Theta}$; let $g(\theta)$ be assigned more weights when we focus more on reducing the expected sample size on these parameter points. As an overall evaluation, the WESS $(g)$ integrates the performances on $\bar{\Theta}$ by weighting the expected sample sizes.

Motivated by Wang et al. [20], we propose operating $S_{1}$ and $S_{2}$ by the 2-SWPRT. Specifically, the stopping and decision rules of $S_{i}(i=1,2)$ by the 2 -SWPRT are

$$
\begin{gathered}
\tau_{i}^{*}=\inf \left\{n \geq 1: R_{n}^{i} \geq A_{i} \text { or } \widetilde{R}_{n}^{i} \geq B_{i}\right), \quad i=1,2, \\
d_{i}=I\left(\tau_{i}^{*}<\infty, R_{\tau_{i}^{*}}^{i} \geq A_{i}\right)+i,
\end{gathered}
$$

where

$$
\begin{array}{r}
R_{n}^{i}=\int_{\theta_{i}^{*}}^{\theta_{2 i}} r_{n}\left(\theta, \theta_{2 i-1}\right) g(\theta) d \theta, \\
\widetilde{R}_{n}^{i}=\int_{\theta_{2 i-1}}^{\theta_{i}^{*}} r_{n}\left(\theta, \theta_{2 i}\right) g(\theta) d \theta, \\
i=1,2,
\end{array}
$$

where $A_{i}$ and $B_{i}(i=1,2)$ are the boundary parameters $(0<$ $\left.A_{i}, B_{i}<\infty\right)$. Hence, the stopping and decision rules of the combined 2-SWPRT are defined as

$$
\begin{gathered}
\tau=\max \left(\tau_{1}^{*}, \tau_{2}^{*}\right), \\
d= \begin{cases}1, & d_{1}=1, d_{2}=2 ; \\
2, & d_{1}=2, d_{2}=2 ; \\
3, & d_{1}=2, d_{2}=3 .\end{cases}
\end{gathered}
$$

Some features of the combined 2-SWPRT are provided in the following theorems, whose proofs are provided in appendices.

First, we show the error probabilities of the combined 2-SWPRT can be easily controlled and the stopping time is finite.

Theorem 1. There exist boundaries $A_{i}$ and $B_{i}(i=1,2)$ such that $(\tau, d)$ in (15) belongs to $\Upsilon(\alpha, \beta)$. 
Theorem 2. For any given nonnegative sectionally continuous weight function $g(\theta)$, the stopping time of the combined 2SWPRT is finite.

Second, we prove that the combined 2-SWPRT is asymptotically optimal on $\bar{\Theta}$.

Definition 3. $(\tau, d) \in \Upsilon(\alpha, \beta)$ is said to be asymptotically optimal on $\bar{\Theta}$, if

$$
\lim _{\substack{\alpha_{i}+\beta_{i} \rightarrow 0 \\ \log \left(\alpha_{i}\right) \approx \log \left(\beta_{i}\right)}} \frac{E_{\theta} \tau}{-\log \left(\alpha_{i}+\beta_{i}\right)}=J_{i}(\theta), \quad \theta \in\left[\theta_{2 i-1}, \theta_{2 i}\right],
$$

where $J_{i}(\theta)=\min \left(1 / K\left(\theta, \theta_{2 i-1}\right), 1 / K\left(\theta, \theta_{2 i}\right)\right), i=1,2$.

Theorem 4. When $A_{i}=\alpha_{i}^{-1}$ and $B_{i}=\beta_{i}^{-1}$, the $(\tau, d)$ defined by (15) is asymptotically optimal on $\bar{\Theta}$.

Third, we show that any positive moment of the stopping time is asymptotically optimal on the indifference-zones.

Theorem 5. Under the conditions of Theorem 4, for all $q \geq 1$ and $\theta \in\left[\theta_{2 i-1}, \theta_{2 i}\right], i=1,2$,

$$
\lim _{\substack{\alpha_{i}+\beta_{i} \rightarrow 0 \\ \log \left(\alpha_{i}\right) \approx \log \left(\beta_{i}\right)}} E_{\theta}\left[\left(\frac{\tau}{-\log \left(\alpha_{i}+\beta_{i}\right)}\right)^{q}\right]=\left(\frac{1}{J_{i}(\theta)}\right)^{q}, \quad i=1,2
$$

\section{Simulation Studies}

In this section, we conduct simulation studies to examine the performances of the combined 2-SWPRT, the SobelWald test, and Whitehead-Brunier test based on the normal and Bernoulli distributions. In particular, we considered two weight functions for $g(\theta)$ as follows; (1) uniform weights: $g(\theta)=0.5 \sum_{i=1}^{2} I\left(\theta \in\left[\theta_{2 i-1}, \theta_{2 i}\right]\right) /\left(\theta_{2 i}-\theta_{2 i-1}\right) ;(2)$ KL weights: $g(\theta)=0.5 \sum_{i=1}^{2} I\left(\theta \in\left[\theta_{2 i-1}, \theta_{2 i}\right]\right) M_{i}(\theta) /\left[\int_{\theta_{2 i-1}}^{\theta_{2 i}} M_{i}(\theta) d \theta\right]$, where $M_{i}(\theta)=\max \left(K\left(\theta, \theta_{2 i-1}\right), K\left(\theta, \theta_{2 i}\right)\right)$. As in Wang et al. [20], the corresponding formulations of the statistics $R_{n}^{i}$ and $\widetilde{R}_{n}^{i}(i=1,2)$ can be obtained. The boundaries of the tests are determined through $10^{6}$ Monte Carlo trials, which make the relative differences between the real error probabilities $\left(\alpha_{i}^{\prime}\right.$ and $\left.\beta_{i}^{\prime}\right)$ and the required ones $\left(\alpha_{i}\right.$ and $\left.\beta_{i}\right)$ within $1 \%$; that is, $\left|\alpha_{i}^{\prime}-\alpha_{i}\right| / \alpha_{i}<1 \%$ and $\left|\beta_{i}^{\prime}-\beta_{i}\right| / \beta_{i}<1 \%$.

Given the boundaries, we obtained the simulated $\operatorname{WESS}(g)=\sum_{\theta \in S} E_{\theta} \tau \cdot g(\theta)$ to approximate integral (12) as follows. Let $\left[\theta_{1}, \theta_{2}\right]$ and $\left[\theta_{3}, \theta_{3}\right]$ be discrete as the finite sets of parameters $S_{1}=\left[\theta_{1}, \theta_{1}+\Delta, \theta_{1}+2 \Delta, \ldots, \theta_{1}^{*}, \ldots, \theta_{2}-\Delta, \theta_{2}\right]$ and $S_{2}=\left[\theta_{3}, \theta_{3}+\Delta, \theta_{3}+2 \Delta, \ldots, \theta_{2}^{*}, \ldots, \theta_{4}-\Delta, \theta_{4}\right]$ with increase $\Delta$, respectively. Denote $S=S_{1} \cup S_{2} \subset \bar{\Theta}$ and the weight function $g(\theta)$ is calculated based on $\theta \in S$; that is, $g(\theta)=0.5 \sum_{i=1}^{2} I\left(\theta \in S_{i}\right) M_{i}(\theta) /\left[\sum_{\theta \in S_{i}} M_{i}(\theta) d \theta\right]$ for $\mathrm{KL}$ weights. We also compute the RMI to assess the relative efficiency between different test plans. According to Wang et al. [20], we define

$$
\operatorname{RMI}(g)=\sum_{\theta \in S} \frac{E_{\theta} \tau-S E_{\theta} \tau}{S E_{\theta} \tau} \cdot g(\theta),
$$

where $S E_{\theta} \tau$ is the smallest $E_{\theta} \tau$ among the compared tests, that is, the Sobel-Wald test, the Whitehead-Brunier test, and the combined 2-SWPRT. A test plan with a smaller RMI $(g)$ value is considered better in its overall performance.

4.1. Test for the Normal Mean with Known Variance. Suppose $X_{1}, X_{2}, \ldots$ are i.i.d. from $N(\theta, 1),-\theta_{1}=\theta_{4}=1.5,-\theta_{2}=\theta_{3}=$ 0.5 , and $\alpha_{1}=\alpha_{2}=\beta_{1}=\beta_{2}=0.01$. According to Lorden [21], we have $\theta_{1}^{*}=-1$ and $\theta_{2}^{*}=1$. The stopping boundaries are obtained as follows:

(1) for the Sobel-Wald test, $A_{i}^{\mathcal{S}}=0.018$ and $B_{i}^{\mathcal{S}}=55.73$;

(2) for the Whitehead-Brunier test, $A_{i}^{w}=B_{i}^{w}=37.36$;

(3) for the combined 2-SWPRT, $A_{i}=B_{i}=26.92$ for the uniform weights and $A_{i}=B_{i}=13.78$ for the KL weights, respectively.

As expected, we found that $\alpha_{13}$ and $\alpha_{31}$ of these three tests are equal to 0 . Set $\Delta=0.05$. Through another simulation study with $10^{5}$ replications, the WESS $(g)$ and $\operatorname{RMI}(g)$ are presented in Table 1. Similarly, the expected sample sizes for $\theta \in[-2,2]$ are illustrated in Figure 1.

It is clear that the combined 2-SWPRTs have the smallest WESS $(g)$ in all cases. In fact, compared with the Sobel-Wald and Whitehead-Brunier tests, the WESS $(g)$ of the combined 2-SWPRT has been reduced by $11.36 \%$ and $5.86 \%$ for the uniform weights, and $8.13 \%$ and $7.57 \%$ for the KL weights. Meanwhile, in terms of the RMI $(g)$, the combined 2-SWPRT also performs best overall.

From Figure 1, it also can be seen that the expected sample size of the combined 2-SWPRT is slightly larger than the Whitehead-Brunier test when the true parameter is close to $\theta_{i}^{*}(i=1,2)$ and almost the same as the Sobel-Wald test when the true parameter is close to $\theta_{2 i-1}$ or $\theta_{2 i}(i=1,2)$. When the true parameter belongs to $\Theta_{k}(k=1,2,3)$, the combined 2-SWPRT performs better than the Whitehead-Brunier test and is comparable with the Sobel-Wald test.

4.2. Test for the True Proportion of a Bernoulli Distribution. Suppose $X_{1}, X_{2}, \ldots$ are i.i.d. random variables from the Bernoulli distribution and $P\left(X_{1}=1\right)=p=1-P\left(X_{1}=\right.$ $0)(0<p<1)$. The three composite hypotheses' testing problem is

$$
\begin{aligned}
& H_{0}: p \leq p_{1} \text { versus } \\
& H_{1}: p_{2} \leq p \leq p_{3} \text { versus } \\
& H_{3}: p \geq p_{4},
\end{aligned}
$$

where $0<p_{1}<p_{2}<p_{3}<p_{4}<1$. Let $\alpha_{1}=\alpha_{2}=\beta_{1}=\beta_{2}=$ $0.01, p_{1}=0.1, p_{2}=0.3, p_{3}=0.4$, and $p_{4}=0.7$. According to (9), we have

$$
p_{i}^{*}=\frac{\log \left(\left(1-p_{2 i-1}\right) /\left(1-p_{2 i}\right)\right)}{\left[\log \left(p_{2 i} / p_{2 i-1}\right)+\log \left(\left(1-p_{2 i-1}\right) /\left(1-p_{2 i}\right)\right)\right]},
$$


TABLE 1: WESS $(g)$ and RMI $(g)$ for testing normal mean.

\begin{tabular}{lcccccc}
\hline$g$ & \multicolumn{3}{c}{ Uniform weights } & \multicolumn{2}{c}{ KL weights } \\
& Sobel-Wald & Whitehead-Brunier & Combined 2-SWPRT & Sobel-Wald & Whitehead-Brunier & Combined 2-SWPRT \\
\hline WESS & 15.231 & 14.342 & $\mathbf{1 3 . 5 1 1}$ & 13.818 & 13.734 & $\mathbf{1 2 . 6 9 4}$ \\
RMI & 0.268 & 0.1652 & $\mathbf{0 . 0 1 8 3}$ & 0.1629 & 0.2117 & $\mathbf{0 . 0 1 7 8}$ \\
\hline
\end{tabular}

TABLE 2: WESS $(g)$ and RMI $(g)$ for testing proportion in Bernoulli distribution.

\begin{tabular}{lcccccc}
\hline$g$ & \multicolumn{3}{c}{ Uniform weights } & \multicolumn{2}{c}{ KL weights } \\
& Sobel-Wald & Whitehead-Brunier & Combined 2-SWPRT & Sobel-Wald & Whitehead-Brunier & Combined 2-SWPRT \\
\hline WESS & 48.978 & 44.163 & $\mathbf{4 3 . 2 5 3}$ & 43.387 & 41.262 & $\mathbf{4 0 . 0 3 5}$ \\
RMI & 0.2508 & 0.0726 & $\mathbf{0 . 0 1 6 3}$ & 0.1615 & 0.0985 & $\mathbf{0 . 0 2 1 5}$ \\
\hline
\end{tabular}

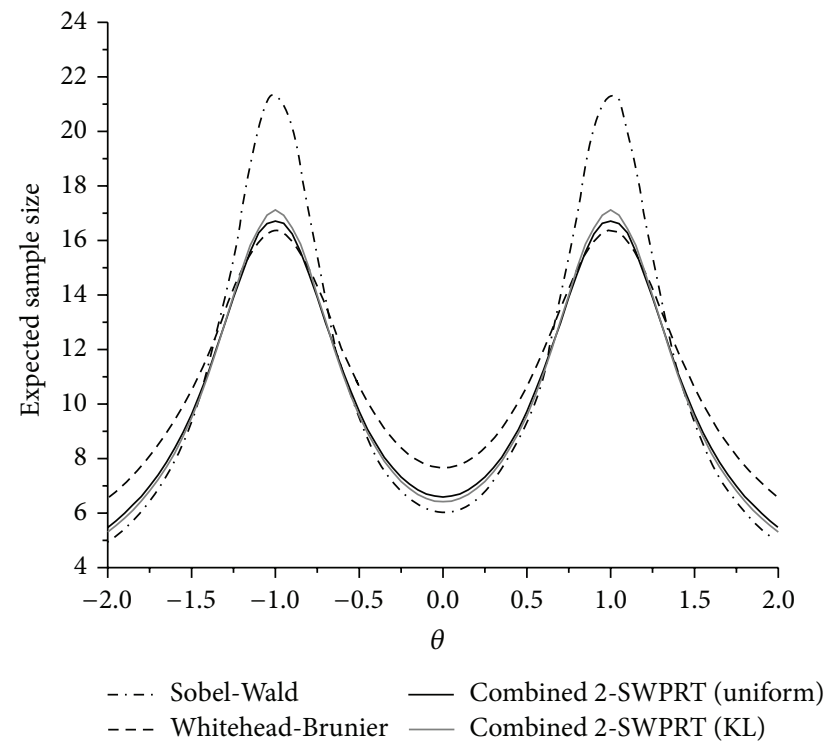

FIGURE 1: Expected sample sizes for testing normal mean, $\alpha_{1}=\alpha_{2}=$ $\beta_{1}=\beta_{2}=0.01,-\theta_{1}=\theta_{4}=1.5$, and $-\theta_{2}=\theta_{3}=0.5$.

such that $p_{1}^{*}=0.186$ and $p_{2}^{*}=0.553$ in the WhiteheadBrunier test and combined 2-SWPRT. The stopping boundaries are obtained as follows:

(1) for the Sobel-Wald test, $A_{1}^{s}=0.012, B_{1}^{s}=66.52, A_{2}^{s}=$ 0.014 , and $B_{2}^{s}=77.23$;

(2) for the Whitehead-Brunier test, $A_{1}^{w}=39.78, B_{1}^{w}=$ 46.51, $A_{2}^{w}=44.33$, and $B_{2}^{w}=43.27$;

(3) for the combined 2-SWPRT, $A_{1}=13.06, B_{1}=20.32$, $A_{2}=16.96$, and $B_{2}=16.27$ for the uniform weights and $A_{1}=19.62, B_{1}=14.35, A_{2}=17.18$, and $B_{2}=$ 13.27 for the KL weights.

In this case, the values of $\alpha_{13}=\alpha_{31}=0$. Set $\Delta=0.0625$. Through another simulation study with $10^{5}$ replications, the WESS $(g)$ and $\operatorname{RMI}(g)$ are presented in Table 2. Similarly, the expected sample sizes for $p \in(0,1)$ are illustrated in Figure 2 .

It can be seen from Table 2 that the combined 2-SWPRT still has the smallest WESS $(g)$ and $\operatorname{RMI}(g)$ for the Bernoulli distribution. Meanwhile, from Figure 2, we have similar

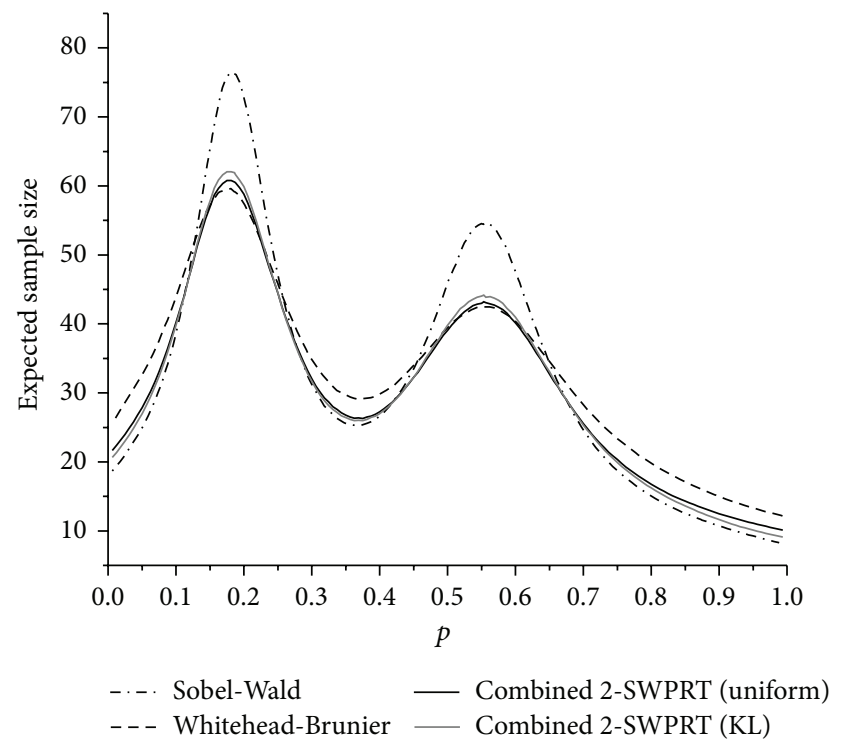

FIGURE 2: Expected sample sizes for testing proportion in Bernoulli distribution, $\alpha_{1}=\alpha_{2}=\beta_{1}=\beta_{2}=0.01, p_{2}=0.1, p_{2}=0.3, p_{3}=0.4$, and $p_{4}=0.7$.

conclusions as those in the normal distribution cases in Section 4.1 .

\section{Summary}

In this paper, we propose the WESS $(g)$ to evaluate the overall performance on the indifference-zones for three composite hypotheses' testing problem. In order to minimize WESS $(g)$ to control the expected sample sizes, we developed a new sequential test by utilizing two 2-SWPRTs simultaneously. We have shown the proposed test is an asymptotically optimal test in the sense of asymptotically minimizing the expected sample sizes on the indifferent-zones.

According to the simulation results, compared with the Sobel-Wald and Whitehead-Brunier tests, we conclude that the proposed test has the following merits: (1) it has the smallest WESS $(g)$ and $\operatorname{RMI}(g)$; (2) when the true parameter is close to $\theta_{i}^{*}(i=1,2)$, the proposed test has comparable performance with Whitehead-Brunier test; when the true parameter is close to $\theta_{2 i-1}$ or $\theta_{2 i}(i=1,2)$, it has almost 
the same results as the Sobel-Wald test; when the true parameter does not belong to $\bar{\Theta}$, the proposed test also performs better than the Whitehead-Brunier test and has comparable performance with the Sobel-Wald test; (3) the proposed test is easy to implement and can be extended to multihypothesis testing problems. Future work will be concerned with the method of determining the boundaries in an analytical way instead of the Monte Carlo method.

\section{Appendix}

We provide sketch proofs of Theorems 1, 2, 4, and 5 .

Proof of Theorem 1. Let $\mathscr{F}_{n}=\sigma\left(x_{1}, \ldots, x_{n}\right), n=1,2, \ldots$ Note that $\left(R_{n}^{1}, F_{n}, n \geq 1\right)$ is a supermartingale under $P_{\theta}, \forall \theta \in \Theta_{1}$. Therefore, for all $\theta \in \Theta_{1}$,

$$
\begin{aligned}
P_{\theta}(d=2) & \leq P_{\theta}\left(\tau_{1}<\infty\right) \\
& \leq \int_{\left\{\tau_{1}<\infty\right\}} A_{1}^{-1} R_{\tau_{1}}^{1} d P_{\theta} \\
& \leq E_{\theta}\left[A_{1}^{-1} R_{1}^{1}\right] .
\end{aligned}
$$

On the other hand, following Lemma 1 of Chen and Hickernell [22], for any positive integer $m$ and $\theta \leq \theta_{1}<\lambda$, we have

$$
E_{\theta}\left[r_{m}\left(\lambda, \theta_{1}\right)\right] \leq 1
$$

Thus,

$$
\begin{aligned}
E_{\theta}\left[A_{1}^{-1} R_{1}^{1}\right] & =A_{1}^{-1} \int_{\theta_{1}^{*}}^{\theta_{2}} E_{\theta}\left[r_{1}\left(t, \theta_{1}\right)\right] g(t) d t \\
& \leq A_{1}^{-1} \int_{\theta_{1}^{*}}^{\theta_{2}} g(t) d t .
\end{aligned}
$$

Combining (A.1) and (A.3), we have

$$
P_{\theta}(d=2) \leq A_{1}^{-1} \int_{\theta_{1}^{*}}^{\theta_{2}} g(t) d t, \quad \forall \theta \in \Theta_{1} .
$$

In particular, setting

$$
A_{1}=\alpha_{1}^{-1} \int_{\theta_{1}^{*}}^{\theta_{2}} g(t) d t
$$

we have $\sup _{\theta \in \Theta_{1}} P_{\theta}(d=2) \leq \alpha_{1}$. Similarly, we can prove that $\sup _{\theta \in \Theta_{2}} P_{\theta}(d=1) \leq \beta_{1}$, $\sup _{\theta \in \Theta_{2}} P_{\theta}(d=3) \leq \alpha_{2}$, and $\sup _{\theta \in \Theta_{3}} P_{\theta}(d=2) \leq \beta_{2}$ with $B_{1}=\beta_{1}^{-1} \int_{\theta_{1}}^{\theta_{1}^{*}} g(t) d t, A_{2}=$ $\alpha_{2}^{-1} \int_{\theta_{2}^{*}}^{\theta_{4}} g(t) d t$, and $B_{2}=\beta_{2}^{-1} \int_{\theta_{3}}^{\theta_{2}^{*}} g(t) d t$, respectively.

Proof of Theorem 2. If $g(\theta)$ is a sectionally continuous function, according to Theorem 3.2 of Wang et al. [20], we know that (1) for all $A_{1}>0$ and $s_{1}>\left(\psi\left(\theta_{1}^{*}\right)-\psi\left(\theta_{1}\right)\right) /\left(\theta_{1}^{*}-\theta_{1}\right)$, there exists $T_{A_{1}}\left(s_{1}\right)<\infty$, such that $R_{n}^{1} \geq A_{1}$ when $n \geq T_{A_{1}}\left(s_{1}\right)$ and $S_{n} \geq n s_{1} ;(2)$ for all $B_{1}>0$ and $s_{1}<\left(\psi\left(\theta_{2}\right)-\psi\left(\theta_{1}^{*}\right)\right) /\left(\theta_{2}-\theta_{1}^{*}\right)$, there exists $T_{B_{1}}\left(s_{1}\right)<\infty$, such that $\widetilde{R}_{n}^{1} \geq B_{1}$ when $n \geq T_{B_{1}}\left(s_{1}\right)$ and $S_{n} \leq n s_{1}$, where $S_{n}=\sum_{l=1}^{n} x_{l}$.
Noting that $\psi(\theta)$ is convex, we have $\left(\psi\left(\theta_{1}^{*}\right)-\psi\left(\theta_{1}\right)\right) /\left(\theta_{1}^{*}-\right.$ $\left.\theta_{1}\right)<\left(\psi\left(\theta_{2}\right)-\psi\left(\theta_{1}^{*}\right)\right) /\left(\theta_{2}-\theta_{1}^{*}\right)$. It is easy to choose $s_{1}$ such that

$$
\frac{\psi\left(\theta_{1}^{*}\right)-\psi\left(\theta_{1}\right)}{\theta_{1}^{*}-\theta_{1}}<s_{1}<\frac{\psi\left(\theta_{2}\right)-\psi\left(\theta_{1}^{*}\right)}{\theta_{2}-\theta_{1}^{*}} .
$$

Let $T_{A_{1} B_{1}}=\max \left(T_{A_{1}}\left(s_{1}\right), T_{B_{1}}\left(s_{1}\right)\right)$. Then, we have $\tau_{1}^{*} \leq T_{A_{1} B_{1}}$. Similarly, for all $A_{2}>0$ and $B_{2}>0$, we can prove that there exist $T_{A_{2}}\left(s_{2}\right)<\infty$ and $T_{B_{2}}\left(s_{2}\right)<\infty$ such that $\tau_{2}^{*} \leq T_{A_{2} B_{2}}$. Thus, we have

$$
\tau \leq \max \left(T_{A_{1} B_{1}}, T_{A_{2} B_{2}}\right) .
$$

Proof of Theorem 4. Using Hoeffding inequality (see Hoeffding [23]), we know

$$
\lim _{\substack{\alpha_{1}+\beta_{1} \rightarrow 0 \\ \log \left(\alpha_{1}\right) \approx \log \left(\beta_{1}\right)}} \frac{E_{\theta} \tau}{-\log \left(\alpha_{1}+\beta_{1}\right)} \geq J_{1}(\theta), \quad \theta \in\left[\theta_{1}, \theta_{2}\right],
$$

so it suffices to show

$$
\lim _{\substack{\alpha_{1}+\beta_{1} \rightarrow 0 \\ \log \left(\alpha_{1}\right) \approx \log \left(\beta_{1}\right)}} \frac{E_{\theta} \tau}{-\log \left(\alpha_{1}+\beta_{1}\right)} \leq J_{1}(\theta), \quad \theta \in\left[\theta_{1}, \theta_{2}\right] .
$$

According to Theorem 3.3 of Wang et al. [20], for all $\theta \in$ $\left[\theta_{1}^{*}, \theta_{2}\right]$

$$
\left.\lim _{\alpha_{1} \rightarrow 0} \frac{\tau_{1}}{\log \left(A_{1}\right)}=\frac{1}{K\left(\theta, \theta_{1}\right)} \quad \text { (a.s. } P_{\theta}\right) .
$$

Since $A_{1}=\alpha_{1}^{-1}$, when $\alpha_{1}+\beta_{1} \rightarrow 0$ and $\log \left(\alpha_{1}\right) \approx \log \left(\beta_{1}\right)$, we have

$$
-\log \left(\alpha_{1}+\beta_{1}\right) \longrightarrow-\log \left(\alpha_{1}\right)=\log \left(A_{1}\right) .
$$

Therefore, for all $\theta \in\left[\theta_{1}^{*}, \theta_{2}\right]$,

$$
\lim _{\substack{\alpha_{1}+\beta_{1} \rightarrow 0 \\ \log \left(\alpha_{1}\right) \approx \log \left(\beta_{1}\right)}} \frac{E_{\theta} \tau_{1}^{*}}{-\log \left(\alpha_{1}+\beta_{1}\right)} \leq \lim _{\alpha_{1} \rightarrow 0} \frac{E_{\theta} \tau_{1}}{\log \left(A_{1}\right)}=\frac{1}{K\left(\theta, \theta_{1}\right)} .
$$

Similarly, for all $\theta \in\left[\theta_{1}, \theta_{1}^{*}\right]$, we have

$$
\lim _{\substack{\alpha_{1}+\beta_{1} \rightarrow 0, \log \left(\alpha_{1}\right) \approx \log \left(\beta_{1}\right)}} \frac{E_{\theta} \tau_{1}^{*}}{-\log \left(\alpha_{1}+\beta_{1}\right)} \leq \lim _{\beta_{1} \rightarrow 0} \frac{E_{\theta} \widetilde{\tau}_{1}}{\log \left(B_{1}\right)}=\frac{1}{K\left(\theta, \theta_{2}\right)} .
$$

Combining two inequalities (A.12) and (A.13), we have

$$
\lim _{\substack{\alpha_{1}+\beta_{1} \rightarrow 0 \\ \log \left(\alpha_{1}\right) \approx \log \left(\beta_{1}\right)}} \frac{E_{\theta} \tau}{-\log \left(\alpha_{1}+\beta_{1}\right)} \leq J_{1}(\theta), \quad \theta \in\left[\theta_{1}, \theta_{2}\right] .
$$

According to (A.8) and (A.14),

$$
\lim _{\substack{\alpha_{1}+\beta_{1} \rightarrow 0 \\ \log \left(\alpha_{1}\right) \approx \log \left(\beta_{1}\right)}} \frac{E_{\theta} \tau}{-\log \left(\alpha_{1}+\beta_{1}\right)}=J_{1}(\theta), \quad \theta \in\left[\theta_{1}, \theta_{2}\right] .
$$


Similarly, we can prove that

$$
\lim _{\substack{\alpha_{2}+\beta_{2} \rightarrow 0 \\ \log \left(\alpha_{2}\right) \approx \log \left(\beta_{2}\right)}} \frac{E_{\theta} \tau}{-\log \left(\alpha_{2}+\beta_{2}\right)}=J_{2}(\theta), \quad \theta \in\left[\theta_{3}, \theta_{4}\right] .
$$

Proof of Theorem 5. Using Lemma 3.6 of Chen [24], for all $q \geq 1$, we know

$$
\begin{aligned}
& \lim _{\alpha_{1} \rightarrow 0} E_{\theta}\left[\left(\frac{\tau_{1}}{-\log \left(\alpha_{1}\right)}\right)^{q}\right]=\frac{1}{\left[K\left(\theta, \theta_{1}\right)\right]^{q}}, \quad\left(\theta_{1}^{*} \leq \theta \leq \theta_{2}\right), \\
& \lim _{\beta_{1} \rightarrow 0} E_{\theta}\left[\left(\frac{\tilde{\tau}_{1}}{-\log \left(\beta_{1}\right)}\right)^{q}\right]=\frac{1}{\left[K\left(\theta, \theta_{2}\right)\right]^{q}}, \quad\left(\theta_{1} \leq \theta \leq \theta_{1}^{*}\right) .
\end{aligned}
$$

Similar to Theorem 4 , for all $\theta \in\left[\theta_{1}^{*}, \theta_{2}\right]$, we have

$$
\begin{aligned}
& \lim _{\substack{\alpha_{1}+\beta_{1} \rightarrow 0 \\
\log \left(\alpha_{1}\right) \approx \log \left(\beta_{1}\right)}} E_{\theta}\left[\left(\frac{\tau_{1}^{*}}{-\log \left(\alpha_{1}+\beta_{1}\right)}\right)\right]^{q} \\
& \leq \lim _{\alpha_{1} \rightarrow 0} E_{\theta}\left[\left(\frac{\tau_{1}}{-\log \left(\alpha_{1}\right)}\right)\right]^{q}=\frac{1}{\left[K\left(\theta, \theta_{1}\right)\right]^{q}} .
\end{aligned}
$$

For all $\theta \in\left[\theta_{1}, \theta_{1}^{*}\right]$, there is

$$
\begin{aligned}
& \lim _{\substack{\alpha_{1}+\beta_{1} \rightarrow 0 \\
\log \left(\alpha_{1}\right) \approx \log \left(\beta_{1}\right)}} E_{\theta}\left[\left(\frac{\tau_{1}^{*}}{-\log \left(\alpha_{1}+\beta_{1}\right)}\right)\right]^{q} \\
& \quad \leq \lim _{\beta_{1} \rightarrow 0} E_{\theta}\left[\left(\frac{\tilde{\tau}_{1}}{-\log \left(\beta_{1}\right)}\right)\right]^{q}=\frac{1}{\left[K\left(\theta, \theta_{2}\right)\right]^{q}} .
\end{aligned}
$$

According to (A.18), (A.19), and Hoeffding inequality, we have

$$
\begin{gathered}
\lim _{\substack{\alpha_{1}+\beta_{1} \rightarrow 0 \\
\log \left(\alpha_{1}\right) \approx \log \left(\beta_{1}\right)}} E_{\theta}\left[\left(\frac{\tau}{-\log \left(\alpha_{1}+\beta_{1}\right)}\right)^{q}\right] \\
=\left(\frac{1}{J_{1}(\theta)}\right)^{q}, \quad \theta \in\left[\theta_{1}, \theta_{2}\right] .
\end{gathered}
$$

Similarly, we can prove that

$$
\begin{gathered}
\lim _{\substack{\alpha_{2}+\beta_{2} \rightarrow 0 \\
\log \left(\alpha_{2}\right) \approx \log \left(\beta_{2}\right)}} E_{\theta}\left[\left(\frac{\tau}{-\log \left(\alpha_{2}+\beta_{2}\right)}\right)^{q}\right] \\
=\left(\frac{1}{J_{1}(\theta)}\right)^{q}, \quad \theta \in\left[\theta_{3}, \theta_{4}\right] .
\end{gathered}
$$

\section{Conflict of Interests}

The authors declare that there is no conflict of interests regarding the publication of this paper.

\section{Acknowledgments}

The authors would like to thank the Academic Editor Antonino Laudani and an anonymous referee for their insightful comments and suggestions on this paper, which have led to significant improvements. This work was supported by the Postdoctoral Science Foundation of China (2014M560317), the National Science Fund of China (11271135, 11471119, 11371142, and 11101156), the Fundamental Research Funds for the Central Universities, the 111 Project (B14019), and the Program of Shanghai Subject Chief Scientist (14XD1401600).

\section{References}

[1] J. J. Goeman, A. Solari, and T. Stijnen, "Three-sided hypothesis testing: simultaneous testing of superiority, equivalence and inferiority," Statistics in Medicine, vol. 29, no. 20, pp. 2117-2125, 2010.

[2] K. S. Fu, Sequential Methods in Pattern Recognition and Learning, Academic Press, New York, NY, USA, 1968.

[3] T. McMillen and P. Holmes, "The dynamics of choice among multiple alternatives," Journal of Mathematical Psychology, vol. 50, no. 1, pp. 30-57, 2006.

[4] J. J. Bussgang, "Sequential methods in radar detection," Proceedings of the IEEE, vol. 58, no. 5, pp. 731-743, 1970.

[5] S. L. Anderson, "Simple method of comparing the breaking strength of two yarns," Journal of the Textle Institute, vol. 45, pp. $472-479,1954$

[6] Y. Li, X. L. Pu, and F. Tsung, "Adaptive charting schemes based on double sequential probability ratio tests," Quality and Reliability Engineering International, vol. 25, no. 1, pp. 21-39, 2009.

[7] I. V. Pavlov, "A sequential procedure for testing many composite hypotheses," Theory of Probability \& Its Applications, vol. 32, no. 1, pp. 138-142, 1988.

[8] C. W. Baum and V. V. Veeravalli, "A sequential procedure for multihypothesis testing," IEEE Transactions on Information Theory, vol. 40, no. 6, pp. 1994-2007, 1994.

[9] V. P. Dragalin, A. G. Tartakovsky, and V. V. Veeravalli, "Multihypothesis sequential probability ratio tests, I: asymptotic optimality," IEEE Transactions on Information Theory, vol. 45, no. 7, pp. 2448-2461, 1999.

[10] V. P. Dragalin, A. G. Tartakovsky, and V. V. Veeravalli, "Multihypothesis sequential probability ratio tests: II. Accurate asymptotic expansions for the expected sample size," IEEE Transactions on Information Theory, vol. 46, no. 4, pp. 1366$1383,2000$.

[11] M. Sobel and A. Wald, "A sequential decision procedure for choosing one of three hypotheses concerning the unknown mean of a normal distribution," Annals of Mathematical Statistics, vol. 20, pp. 502-522, 1949.

[12] P. Armitage, "Sequential analysis with more than two alternative hypotheses, and its relation to discriminant function analysis," Journal of the Royal Statistical Society. Series B. Methodological, vol. 12, pp. 137-144, 1950.

[13] G. Simons, "Lower bounds for average sample number of sequential multihypothesis tests," Annals of Mathematical Statistics, vol. 38, pp. 1343-1364, 1967. 
[14] G. Lorden, "Likelihood ratio tests for sequential $k$-decision problems," Annals of Mathematical Statistics, vol. 43, pp. 1412$1427,1972$.

[15] J. Whitehead and H. Brunier, "The double triangular test: a sequential test for the two-sided alternative with early stopping under the null hypothesis," Sequential Analysis: Design Methods \& Applications, vol. 9, no. 2, pp. 117-136, 1990.

[16] Y. Li and X. Pu, "Hypothesis designs for three-hypothesis test problems," Mathematical Problems in Engineering, vol. 2010, Article ID 393095, 15 pages, 2010.

[17] Y. Li and X. L. Pu, "A method for designing three-hypothesis test problems and sequential schemes," Communications in Statistics-Simulation and Computation, vol. 39, no. 9, pp. 16901708, 2010.

[18] V. P. Dragalin and A. A. Novikov, "Adaptive sequential tests for composite hypotheses," Surveys of Applied and Industrial Mathematics, vol. 6, pp. 387-398, 1999.

[19] T. L. Lai, "Sequential multiple hypothesis testing and efficient fault detection-isolation in stochastic systems," IEEE Transactions on Information Theory, vol. 46, no. 2, pp. 595-608, 2000.

[20] L. Wang, D. D. Xiang, X. L. Pu, and Y. Li, "A double sequential weighted probability ratio test for one-sided composite hypotheses," Communications in Statistics. Theory and Methods, vol. 42, no. 20, pp. 3678-3695, 2013.

[21] G. Lorden, "2-SPRT's and the modified Kiefer-Weiss problem of minimizing an expected sample size," The Annals of Statistics, vol. 4, no. 2, pp. 281-291, 1976.

[22] J. D. Chen and F. J. Hickernell, "A class of asymptotically optimal sequential tests for composite hypotheses," Science in China Series A, vol. 37, no. 11, pp. 1314-1324, 1994.

[23] W. Hoeffding, "Lower bounds for the expected sample size and the average risk of a sequential procedure," Annals of Mathematical Statistics, vol. 31, pp. 352-368, 1960.

[24] J. D. Chen, "Asymptotic optimality for one class of truncated sequential tests," Science in China. Series A. Mathematics, Physics, Astronomy, vol. 30, pp. 30-41, 1991. 


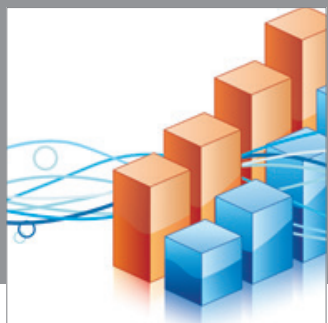

Advances in

Operations Research

mansans

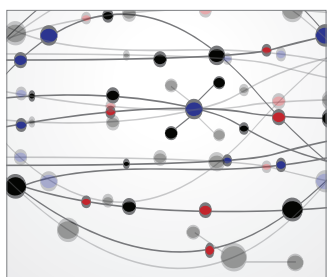

The Scientific World Journal
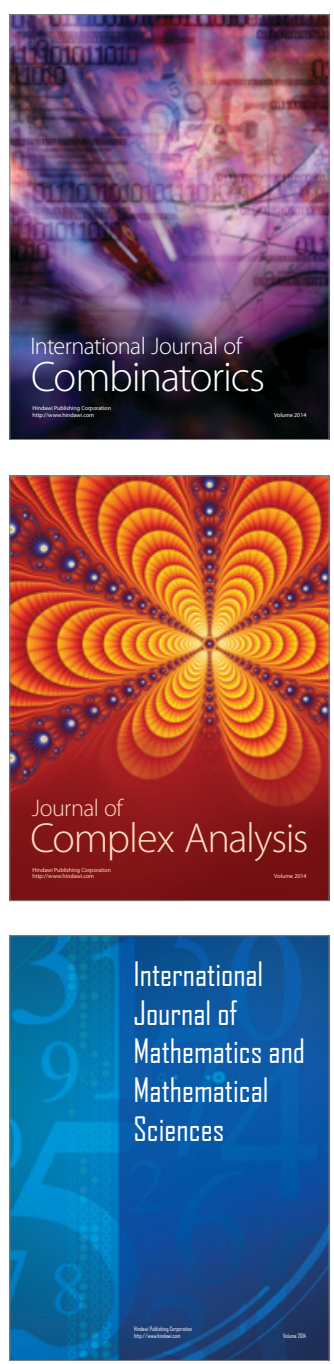
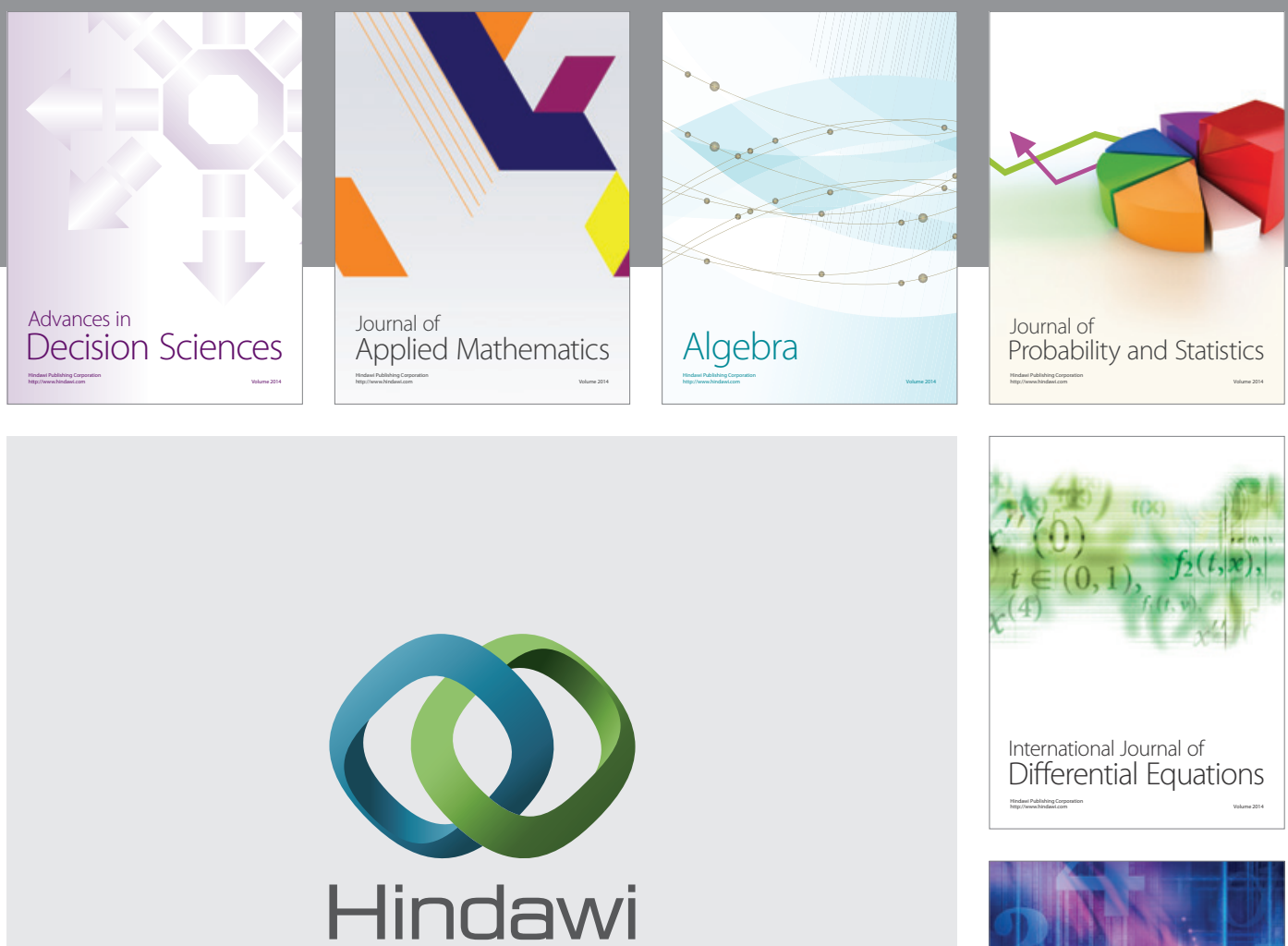

Submit your manuscripts at http://www.hindawi.com
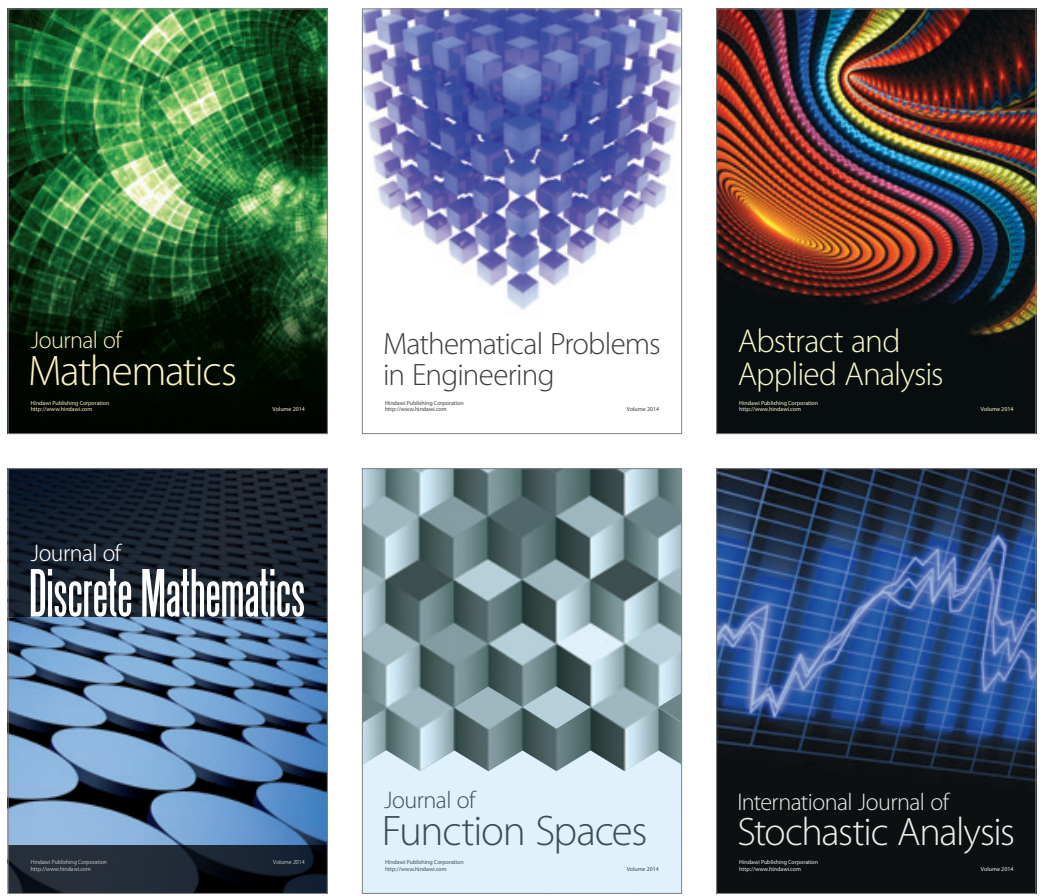

Journal of

Function Spaces

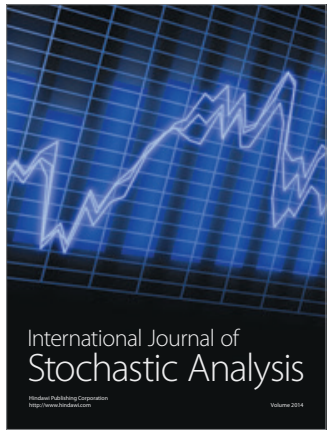

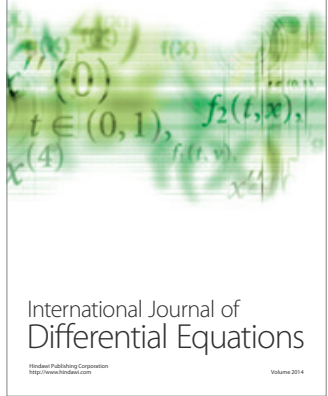
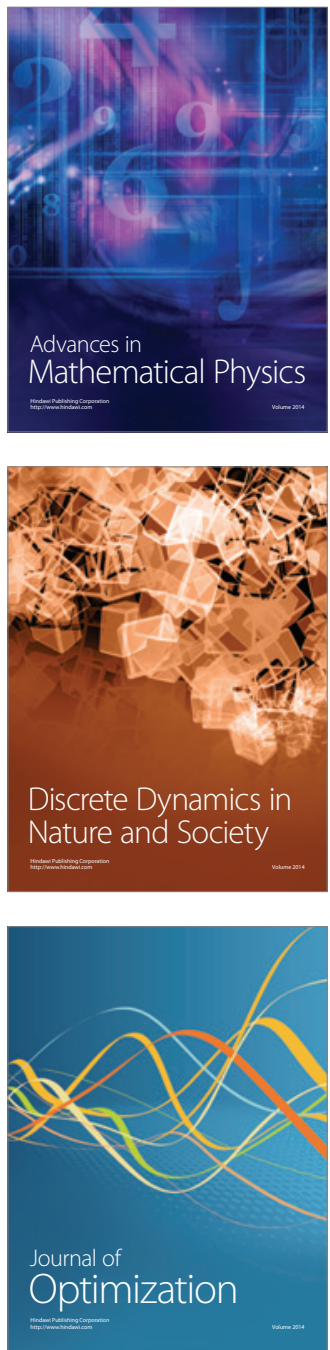